\title{
Correction to \\ An Isomorphism Theorem for Henselian Algebraic Extensions of Valued Fields
}

\author{
Serban A. Basarab - Franz-Viktor Kuhlmann
}

In the example on page 123 of the article [1], the correct equation for $\pi$ should be $\pi^{p-1}=-p$. As a consequence of the forgotten minus sign, the succeeding choice of the polynomial $f$ and the corresponding verification is false. The intention was to provide a non-cyclic extension of $Q_{p}(\varepsilon)$ of degree $p$, where $\varepsilon \neq 1$ denotes a $p$-th root of unity. The polynomial given in our example may be replaced by the polynomial $X^{p}+\pi X+\pi$. Let $a$ be a root of this polynomial and let $\mathbf{K}=\left(Q_{p}(\varepsilon, x), v\right)$ as in the example. Then $v a=\frac{1}{p} v \pi \notin v K$ and consequently, the extension $\mathbf{K}(a) \mid \mathbf{K}$ is purely ramified; the residue field $\overline{K(a)}$ is equal to $I F_{p}$. Moreover, $a$ is the only root of $f$ in $K(a)$ (hence $K(a) \mid K$ is not cyclic). Indeed, assume that $b \neq a$ is another root of $f$. Then the element $z=\frac{b-a}{a}$ satisfies the equation

$$
z^{p-1}+\sum_{i=0}^{p-2}\left(\begin{array}{c}
p \\
i+1
\end{array}\right) z^{i}+\frac{\pi}{a^{p-1}}=0
$$

All binomial coefficients $\left(\begin{array}{c}p \\ i+1\end{array}\right)$ are divisible by $p$ for $0 \leq i \leq p-2$. Moreover, $v \pi a^{-(p-1)}=p v a-(p-1) v a=v a<v p$. Consequently, $v z=\frac{1}{p-1} v a$ which is not an element of $v K(a)$ since $p>2$. This shows that $b \notin K(a)$.

We are very endebted to $\mathrm{F}$. Lorenz for giving us the idea how to find the right polynomial. His argument was as follows. Given a polynomial $f$, its discriminant is by definition a square in its splitting field. So we have to find an irreducible polynomial of degree $p$ over $\varphi_{p}(\varepsilon)$ whose discriminant is not a square in $Q_{p}(\varepsilon)$. Indeed, since the extension by one of its roots $a$ is of odd degree $p$, this discriminant will still not be a square in $Q_{p}(\varepsilon, a)$, showing that $Q_{p}(\varepsilon, a)$ is not the splitting field of $f$. This yields that the extension $Q_{p}(\varepsilon, a) \mid Q_{p}(\varepsilon)$ is not cyclic. 
For polynomials of the form $X^{n}+c X+d$, the discriminant may be computed by the formula

$$
\operatorname{discr} f=(-1)^{n(n-1) / 2}\left(n^{n} d^{n-1}+(1-n)^{n-1} c^{n}\right)
$$

(cf. Lorenz [2], p. 316). With this formula, one finds that the discriminant of $X^{p}+\pi X+\pi$ is equal to $\pm\left(p^{p} \pi^{p-1}+(1-p)^{p-1} \pi^{p}\right)$. So $v \operatorname{discr} f=p v \pi$ which is not divisible by 2 in $v Q_{p}(\varepsilon)$ and $v K$ since $v \pi$ is the smallest element in both value groups.

Later in the same example, there is a paragraph which should better read as follows: "For every element $b \in L$ there exists some $\nu \in\left\{0, \ldots, p^{2}-1\right\}$ and $c \in K$ such that $b^{-1} d_{1}^{\nu} c$ is a 1 -unit, i.e. an element of $1+\mathcal{M}_{L}$. Since $\pi_{\delta}^{*}\left(1+\mathcal{M}_{L}\right) \subset$ $\Theta_{\delta}^{\times}\left(\mathcal{O}_{\mathbf{L}}^{2 \delta}\right)^{\times}$, this shows that $\left(\pi_{\delta}^{*} b\right) H=\left(\pi_{\delta}^{*} d_{1}^{\nu}\right) H . "$

Finally, the compactness argument in Lemma 2.6 should be formulated in a slightly different way: "Since $L \mid K$ is algebraic, we have $L=\bigcup_{i \in I} K_{i}$ where $K_{i} \mid K$ runs through all finite subextensions of $L \mid K$. If every $K_{i}$ admits a $K$-embedding $\sigma_{i}$ in $F$ then there is a $K$-embedding $\sigma$ of $L$ in $F$ such that

$$
\forall i \in I:\left.\quad \sigma\right|_{K_{i}}=\left.\sigma_{j}\right|_{K_{i}} \text { for some } j \in I \text { such that } K_{i} \subset K_{j} .
$$

In particular, if all $\sigma_{i}$ are valuation preserving, then so is $\sigma . "$

\section{References}

[1] Basarab, S. - KUHLMANN, F.-V.: An Isomorphism Theorem for Henselian Algebraic Extensions of Valued Fields. manuscripta math. 77, $113-126(1992)$

[2] Lorenz, F.: Einführung in die Algebra, Teil I. BI-Wiss.-Verl., Mannheim - Wien -Zürich (1987)

Serban A. Basarab

Institute of Mathematics

of the Romanian Academy

P.O.Box 1 - 764

RO - 70700 Bucharest, Romania
Franz-Viktor Kuhlmann

Mathematisches Institut

Universität Heidelberg

Im Neuenheimer Feld 288

D - 6900 Heidelberg, Germany 